(1D) Suzi Hellen Anaice de Araujo ${ }^{1}$

(D)Aldair da Silva Guterres ${ }^{2}$

(DSocorro Nazaré Araújo Almeida Barbosa $^{2}$

(1D) Priscila Matos de Pinho ${ }^{3}$

(D) Rosileide de Souza Torres²

(1) Jessika Larrisa Soares Marinho 4

(10) Mara Ludmila Lima Pereira 4

(D) Nathalia da Silva Reis ${ }^{4}$

(DPaulo de Tarso Toscano Junior ${ }^{2}$

1 Centro Universitário do Estado do Pará, Curso de Pós-Graduação em Nutrição Clínica, Belém, PA,

Brasil.

${ }^{2}$ Fundação Pública Estadual Hospital de Clínicas Gaspar Vianna, Serviço de Nutrição,

Belém, PA, Brasil.

3 Fundação Pública Estadual Hospital de Clínicas Gaspar Vianna, Serviço de Nutrição e Dietética, Belém, PA, Brasil.

${ }^{4}$ Escola Superior da Amazônia, Belém, PA, Brasil.

Correspondência

Suzi Hellen Anaice

suzi_anaice@hotmail.com

\section{Aplicação da triagem de risco nutricional em crianças e adolescentes hospitalizados com cardiopatia congênita}

\author{
Nutritional risk screening application in hospitalized children \\ and adolescents with congenital heart disease
}

\section{Resumo}

Introdução: A triagem nutricional em crianças é de extrema importância no ambiente hospitalar, pois é uma ferramenta simples e eficiente. Objetivo: Detectar o risco nutricional, a partir do método de triagem nutricional pediátrica Strong Kids, em crianças e adolescentes com cardiopatias congênitas. Método: Foram avaliados 81 crianças e adolescentes com cardiopatia congênita, até 48 horas de internação, no período de agosto de 2016 a maio de 2017. Dados foram coletados através de triagem nutricional pediátrica. A análise estatística se deu através do teste qui-quadrado, a fim de verificar o nível de significância, $p<0,05$. Resultados: Dentre os resultados obtidos, houve maior prevalência de crianças com cardiopatia congênita internadas do gênero feminino 60,49\% (n=49). De acordo com a classificação das fases de vida, a predominância ocorreu na fase pré-escolar 46,91\%, seguida de lactentes 27,16\%, adolescentes 13,58\% e escolar 12,35\%. Não houve déficit nutricional para 74,07\%. Segundo o teste qui-quadrado, observou-se relevância do médio risco nutricional. Conclusão: A triagem nutricional pediátrica é fundamental na prática clínica, e sua melhor forma de avaliação é a intervenção nutricional precoce. Isto implicará um tratamento nutricional adequado, em especial para crianças com cardiopatia congênita.

Palavras-chave: Cardiopatias congênitas. Pediatria. Desnutrição.

\begin{abstract}
Introduction: The nutritional screening in children is extremely important in the hospital environment because it is a simple and efficient tool. Objective: Detecting the nutritional risk, from pediatric nutritional screening Strong Kids method in children and adolescents with congenital heart desease. Method: 81 children and adolescents were evaluated with congenital heart desease until 48 hours of hospitalization, in the period from August 2016 to May 2017. The data collection was through a pediatric nutritional screening. The statistical analysis was through chi-square test for the purpose of verify the level of significance, $p<0,05$. Results: Among the results obtained, there was a higher prevalence of children with hospitalized congenital heart desease of the female gender $60,49 \%(n=49)$. According to the classification of the life phases, the highest predominance was in the pre school phase $(46,91 \%)$, then infants $(27,16 \%)$, adolescents (13,58\%) and scholar (12,35\%). There was no nutritional deficit for 74,07\%. According chi-square test, it was observed a relevance to the medium nutritional risk. Conclusion: The pediactric nutritional screening is extremely important in clinical practice, in view of the best form of evaluation through the reach of early nutritional intervention, this will entail adequate nutritional treatment, specially children with congenital heart desease.
\end{abstract}

Keywords: Congenital heart desease. Pediatrics. Malnutrition.. 


\section{INTRODUÇÃO}

A cardiopatia congênita (CC) é definida como uma anormalidade na estrutura e função cardiocirculatória, que está presente desde o nascimento e em alguns casos é descoberta após vários anos. Essas alterações geralmente resultam de alterações no período embrionário e no desenvolvimento fetal defeituoso, sendo consideradas umas das mais sérias doenças crônicas em crianças. ${ }^{1}$ A etiologia dessa patologia ainda não foi totalmente elucidada, mas há alguns fatores de risco relacionados, como fatores genéticos, síndromes cromossômicas, tabagismo, drogas ilícitas e etilismo na gestação, entre outros.²

As crianças portadoras dessa cardiopatia apresentam, na maioria das vezes, dificuldade para se alimentar, além da sudorese excessiva. A alimentação no seio materno é uma grande dificuldade para esses pacientes, pois acabam fazendo um grande esforço para sugar e por isso deixam de mamar, por se cansarem com mais facilidade. Além disso, há uma frequente redução do apetite e diminuição na absorção dos nutrientes. Esses fatores contribuem para um desequilíbrio nutricional. ${ }^{3}$

A desnutrição nessas crianças causa um problema hospitalar considerável, pois aumenta o período de internação e, consequentemente, eleva o custo de hospitalização. Portanto, é de extrema importância que seja realizada a triagem nutricional, para que se possa intervir com segurança nos cuidados na evolução clínica e nutricional dos pacientes. ${ }^{4}$ Além disso, é um método de grande relevância no ambiente hospitalar, por ser uma ferramenta simples, de baixo custo e eficiente. ${ }^{5}$

Conforme o exposto, o objetivo deste trabalho é aplicar a triagem nutricional em crianças e adolescentes com cardiopatia congênita internados em um hospital-escola de referência em cardiologia em Belém-PA.

\section{MÉTODOS}

\section{Modelo de estudo e participantes}

Estudo de caráter quantitativo, descritivo e transversal com amostra por conveniência, constituída por 81 crianças e adolescentes portadores de cardiopatia congênita na faixa etária de 2-12 anos, de ambos os sexos, internadas nas primeiras 48 horas de internação na clínica pediátrica da Fundação Pública Estadual Hospital de Clínicas Gaspar Vianna em Belém-PA (FPEHCGV), no período de agosto de 2016 a maio de 2017.

Foram considerados critérios de inclusão: ter idade entre 2-12 anos; possuir diagnóstico de cardiopatia congênita; estar hospitalizado na FPEHCGV; aceitar participar da pesquisa e assinar o termo de consentimento livre e esclarecido (TCLE) e o de assentimento, quando este se fizer necessário. Os critérios de exclusão foram: ter idade < 2 anos e > 12 anos; impossibilidade ou negação em participar da pesquisa e assinar o TCLE; e não estar apto física e mentalmente para ser avaliado; e portadores de síndrome de Down.

No que diz respeito ao critério de exclusão relacionado à idade, justifica-se que a idade máxima de internação na clínica pediátrica da FPEHCGV é de 12 anos, e a mínima está relacionada à aplicabilidade de um dos métodos de triagem nutricional, que não pode ser aplicado em menores de dois anos. Como se trata de pesquisa cujo principal objetivo é a aplicação de método de triagem nutricional, se faz necessária a equivalência em idades dos pacientes envolvidos.

Foi aplicado o método subjetivo de triagem nutricional pediátrica Strong Kids, para avaliar o risco nutricional das crianças. Esse método consiste em um instrumento que apresenta quatro itens de avaliação, a saber: avaliação clínica subjetiva, doença de alto risco, ingestão alimentar e perda de peso. A cada item é dada uma pontuação, fornecida quando a resposta à pergunta for positiva. A somatória desses pontos 
identifica o risco de desnutrição, permitindo ao aplicador decidir sobre a intervenção e o acompanhamento necessário. ${ }^{6}$

A garantia dos aspectos éticos foi pautada segundo os preceitos da Declaração de Helsinque e do Código de Nuremberg, respeitando as normas de pesquisa envolvendo seres humanos (Resolução CNS nº 466/2012) do Conselho Nacional de Saúde. Garantiu-se o sigilo no que se refere à identificação do paciente, mediante autorização do hospital, por meio do Termo de Consentimento Livre e Esclarecido (TCLE), que foi apresentado em duas vias e entregue uma cópia ao responsável, após aprovação do Comitê de Ética em Pesquisa (CEP) da FPEHCGV, conforme o parecer 1.475.974.

\section{Análise Estatística}

A análise estatística foi realizada por meio do software Bioestat 5.0, através do teste qui-quadrado, a fim de verificar o nível de significância entre os mesmos, sendo considerado estatisticamente significativo quando $p<0,05$.

\section{RESULTADOS}

Foram avaliados 81 crianças e adolescentes cardiopatas internados na clínica pediátrica, sendo que $60,49 \%(n=49)$ eram do gênero feminino e 39,51\% masculino $(n=32)$. A maioria encontra-se classificada na fase pré-escolar, com 46,91\%, o que corresponde à faixa etária de 2-6 anos, em seguida lactentes com $27,16 \%$, adolescente com 13,58\% e escolar com 12,35\% (tabela 1).

Tabela 1. Percentual de crianças e adolescentes com cardiopatia congênita internados em um hospital de referência em cardiologia, por gênero e fase de vida. Belém-PA, 2017.

\begin{tabular}{llcc}
\hline Variável & Categoria & N & $\%$ \\
\hline \multirow{3}{*}{ Gênero } & Feminino & 49 & 60,49 \\
& Masculino & 32 & 39,51 \\
& Total & 81 & 100,00 \\
\hline \multirow{3}{*}{ Fase da Vida } & Lactentes & 22 & 27,16 \\
& Pré-Escolar & 38 & 46,91 \\
& Escolar & 10 & 12,35 \\
& Adolescente & 11 & 13,58 \\
& Total & 81 & 100,00 \\
\hline
\end{tabular}

A tabela 2 mostra os itens da avaliação do Strong Kids: Avaliação Clínica Subjetiva, na qual se avalia se há ou não déficit nutricional, constatou que em 74,07\% (n=60) não houve presença déficit nutricional. Neste mesmo item foram identificados 76,19\% ( $n=16)$ de crianças com face crônica, seguidas por 23,81\% (n=5) com déficit de gordura subcutânea.

Tabela 2. Crianças e adolescentes com cardiopatia congênita internados em um hospital de referência em cardiologia, segundo os itens da Triagem de Risco Nutricional Strong Kids. Belém-PA, 2017.

\begin{tabular}{clcc}
\hline Variável & Categoria & N & $\%$ \\
\hline \multirow{3}{*}{ Déficit Nutricional } & Não & 60 & 74,07 \\
& Sim & 21 & 25,93 \\
& Total & 81 & 100,00 \\
\hline
\end{tabular}


Tabela 2. Crianças e adolescentes com cardiopatia congênita internados em um hospital de referência em cardiologia, segundo os itens da Triagem de Risco Nutricional Strong Kids. Belém-PA, 2017.(Cont)

\begin{tabular}{|c|c|c|c|}
\hline Variável & Categoria & $\mathrm{N}$ & $\%$ \\
\hline \multirow{4}{*}{ Sinal de déficit nutricional } & Redução de gordura subcutânea & 5 & 23,81 \\
\hline & Face emagrecida & 16 & 76,19 \\
\hline & Outro sinal & 0 & 0,00 \\
\hline & Total & 21 & 100,00 \\
\hline \multirow{3}{*}{$\begin{array}{l}\text { Presença de doença com alto risco } \\
\text { nutricional ou cirurgia de grande porte }\end{array}$} & Não & 0 & 0,00 \\
\hline & $\operatorname{sim}$ & 81 & 100,00 \\
\hline & Total & 81 & 100,00 \\
\hline \multirow{3}{*}{$\begin{array}{l}\text { Redução da ingestão alimentar ou perdas } \\
\text { nos últimos dias }\end{array}$} & Não & 65 & 80,25 \\
\hline & $\operatorname{sim}$ & 16 & 19,75 \\
\hline & Total & 81 & 100,00 \\
\hline \multirow{6}{*}{$\begin{array}{l}\text { Sinal de redução da ingestão alimentar ou } \\
\text { perdas nos últimos dias }\end{array}$} & Dificuldade de alimentar devido à dor & 0 & 0,00 \\
\hline & Intervenção nutricional prévia & 1 & 6,25 \\
\hline & Vômitos & 1 & 6,25 \\
\hline & Diarreia $>5$ Dias & 2 & 12,50 \\
\hline & Diminuição da ingestão alimentar & 12 & 75,00 \\
\hline & Total & 16 & 100,00 \\
\hline \multirow{3}{*}{$\begin{array}{l}\text { Perda de peso ou ganho insuficiente nas } \\
\text { últimas semanas }\end{array}$} & Não & 54 & 66,67 \\
\hline & Sim & 27 & 33,33 \\
\hline & Total & 81 & 100,00 \\
\hline \multirow{3}{*}{$\begin{array}{l}\text { Sinal de perda de peso ou ganho insuficiente } \\
\text { nas últimas semanas }\end{array}$} & $\begin{array}{l}\text { Não ganho de peso - menos que } 1 \\
\text { ano }\end{array}$ & 10 & 38,46 \\
\hline & Perda de peso- maior que 1 ano & 16 & 61,54 \\
\hline & Total & 26 & 100,00 \\
\hline
\end{tabular}

O segundo item da avaliação pediátrica, sobre a presença de doença de alto risco nutricional ou cirurgia de grande porte, constatou que a totalidade (100\%) das crianças com cardiopatia congênita avaliada necessita realizar a cirurgia cardíaca, que é considerada de grande porte.

Com relação ao terceiro item da avaliação subjetiva pediátrica, que diz respeito à redução de ingestão alimentar ou perdas após o período de internação, 19,75\% ( $n=16)$ apresentaram essas alterações; a diminuição da ingestão alimentar resultou em 80,25\% (n=65). Sinal de redução de ingestão alimentar ou perdas nos últimos dias: diminuição da ingestão alimentar foi de 75\%; diarreia em 12,50\%; vômitos e intervenção nutricionais foram de 6,25\%.

Tais alterações são mais preocupantes por se tratar de crianças cardiopatas em pré e pós-operatório de cirurgia de grande porte, o que pode acarretar emagrecimento, desidratação, dificuldade de absorção devido à perda das microvilosidades intestinais e estado de nutrição inadequado (má nutrição), que não deixam o paciente apto a realizar a cirurgia. Pode haver ainda consequências pós-cirúrgicas, como piora no quadro clínico, dificuldade de cicatrização e até mesmo o óbito.

No que se refere ao quarto item de triagem pediátrica, que avalia a perda de peso ou ganho insuficiente nas últimas semanas ou meses, em 66,67\% ( $n=54$ ) houve perda de peso, e em 33,33\% ( $n=27)$ não houve perda de peso. 
Quanto ao nível do risco nutricional (tabela 3), observou-se que essa população apresentou médio risco nutricional; ou seja, a avaliação subjetiva Strong Kids é viável para a realização de uma triagem nutricional pediátrica, pois é útil para o rastreamento do risco de desnutrição de forma rápida e prática, além de permitir a intervenção precoce.

Tabela 3. Crianças e adolescentes com cardiopatia congênita internados em um hospital de referência em cardiologia, segundo o nível de risco nutricional da Strong Kids. Belém-PA, 2017.

\begin{tabular}{lccc}
\hline Nível de risco nutricional & $n$ & $\%$ & $p$ \\
\hline Alto & 17 & 20,99 & \\
Médio & 64 & 79,01 & $<0.0001$ * \\
Baixo & 0 & 0,00 & \\
\hline Total & 81 & 100,00 & \\
\hline
\end{tabular}

Nota: *Qui-quadrado: $p<0,05$ - diferenças significativas.

\section{DISCUSSÃO}

Após a análise dos dados, observou-se que a maior parte das crianças internadas era do gênero feminino, dados que corroboram o estudo realizado por Aragão, ${ }^{7}$ com 300 crianças cardiopatas internadas no Hospital do Coração em Aracaju. Boeira, ${ }^{8}$ em revisão sistemática da literatura, também constatou maior prevalência do gênero feminino entre os indivíduos avaliados, mas Secker \& Jeejeebhoy ${ }^{9}$ verificaram que 57\% das crianças foram do gênero masculino, no estudo que avaliou 180 crianças a partir do Strong Kids.

Os pacientes do presente estudo foram divididos em grupos por fase da vida para melhor classificação; sendo assim, prevaleceram a fase pré-escolar $(46,91 \%)$ e lactentes $(27,16 \%)$, resultado semelhante ao encontrado na pesquisa de Simões, ${ }^{10}$ com prevalência de pré-escolar (26\%), escolar (24\%) e adolescentes (12,3\%). No entanto, na tese de mestrado de Gouveia, ${ }^{11}$ observou-se predominância na fase de vida préescolar (48,6\%), lactentes (23,1\%) e escolar (28,3\%). Já no estudo de Lama et al., ${ }^{12}$ a amostra foi composta de 103 meninas e 147 meninos na fase pré-escolar. Portanto, os resultados foram semelhantes ao presente estudo. No estudo de Aragão, ${ }^{7}$ também se obteve a maior parte dos pacientes na fase lactente $47 \%$ e préescolar 24\%, o que pode indicar que nessa faixa de vida o período de sinais clínicas da patologia seja mais evidente, pois muitos pacientes apresentam sintomas.

Quanto ao observado na tabela 2, no presente estudo não houve déficit nutricional (74,04\%). De acordo com Monteiro, ${ }^{13}$ crianças nascidas cardiopatas são consideradas um grupo de alto risco nutricional, pois acontece perda de massa corporal, que prejudica o organismo de forma geral, sobretudo o coração e os músculos respiratórios, afetando a função miocárdica e ventilatória.

O estudo desenvolvido por Araújo, ${ }^{14}$ que buscou identificar a incidência de déficit nutricional pediátrico, constatou elevada prevalência nas regiões Norte e Nordeste, que apresentam grande população de baixa renda, um dos fatores responsáveis pela elevação dos índices de morbimortalidade neonatal.

Percebe-se na tabela 2, que 76,19\% dos pacientes apresentam face caquética e $23,81 \%$, redução de gordura subcutânea. Verificou-se que a maioria dos pacientes tem alteração nutricional, já que a patologia de forma geral resulta em muitas alterações nutricionais e psicológicas nos pacientes acometidos com cardiopatia congênita. De acordo com a tese de mestrado de Monteiro, ${ }^{13}$ que analisou o estado nutricional de crianças com cardiopatia congênita, as evidências clínicas promovem desnutrição, como repouso 
prolongado, que diminui a massa muscular; anorexia causada pela patologia e suas comorbidades, hipóxia e hipermetabolismo, responsáveis pela diminuição de peso e piora do prognóstico desses pacientes. 0 estudo realizado por Ribeiro et al.,15 avaliando crianças e adolescentes internados em um hospital privado de Salvador-BA, informou que a desnutrição hospitalar é uma adversidade de saúde pública, e que é importante a intervenção nutricional nessa população, a ser feita precocemente.

Quanto às causas de risco nutricional e desnutrição, observado na tabela 2, 75\% apresentaram diminuição da ingestão alimentar; 12,50\% diarreia > 5 dias; 6,25\% intervenção nutricional prévia e vômitos. Estudo realizado por Teixeira ${ }^{16}$ analisou o perfil nutricional de crianças e adolescentes com cardiopatia congênita e observou que a desnutrição nesses indivíduos se deve a vários fatores: ingestão calórica inadequada, redução de apetite, idade da correção cirúrgica, hipermetabolismo, alterações pulmonares, taquipineia e restrição de volume alimentar.

Quando se avaliou o nível de risco nutricional a partir do presente estudo, constatou-se que 79,01\% dos pacientes avaliados estavam com médio risco nutricional e 20,99\%, alto risco nutricional. Porém, na tese de mestrado de Moutinho, ${ }^{17}$ foram analisadas 104 crianças cardiopatas internadas no Hospital de Coimbra, em que $8,7 \%$ dos pacientes estavam em alto risco nutricional, 58,7\% dos pacientes apresentaram médio risco nutricional, e 32,7\%, baixo risco nutricional. Este resultado não é coerente com o encontrado no presente estudo, até porque se trata de pacientes diferentes.

No entanto, o estudo de Vallandro, ${ }^{18}$ que efetuou a triagem nutricional em 455 crianças internadas com cardiopatia congênita no Hospital da Criança Santo Antônio, observou que 27,3\% das crianças apresentaram risco nutricional baixo, 64,8\% médio risco nutricional e 7,9\% alto risco nutricional. Esse resultado é semelhante ao presente estudo, que verificou a predominância de médio risco nutricional. A pesquisa de Marginean ${ }^{19}$ avaliou 271 crianças com malformação congênita, afecção respiratória e fissura labial ou palatina hospitalizadas, utilizando a triagem nutricional Strong Kids, observando que 63,1\% estavam sem desnutrição ou risco nutricional, 21,8\% em moderado risco nutricional e 15,1\% em alto risco nutricional. Com o comparativo dos estudos, compreende-se que no público da pesquisa, quando se utiliza o Strong Kids como ferramenta de triagem nutricional, a maioria apresentou médio e alto risco nutricional e nenhum paciente em baixo risco nutricional. Portanto, a triagem nutricional é uma ferramenta eficaz e sensível no diagnóstico do risco nesses pacientes.

\section{CONCLUSÃO}

A pesquisa em questão tem como problema central crianças com cardiopatia congênita que apresentaram de moderado a elevado risco nutricional, segundo a análise estatística. Isso se torna preocupante, pois as alterações nutricionais são ocasionadas pelos fatores relacionados com o processo de emagrecimento inerente à fisiopatologia da doença.

De acordo com o processamento estatístico e o levantamento bibliográfico do estudo, constatou-se que a maioria dos pacientes estudados foi do gênero feminino e a fase de vida mais predominante foi préescolar e lactentes.

Assim, a triagem nutricional é de fundamental importância na prática clínica, pois tendo em vista a melhor forma de avaliação através do alcance de uma intervenção nutricional precoce, isto implicará a necessidade de um tratamento nutricional adequado, em especial as crianças com cardiopatia congênita. 


\section{AGRADECIMENTOS}

Ao Comitê de Ética e Pesquisa da Fundação Pública Estadual Hospital de Clínicas Gaspar Vianna (FPEHCGV), por ter aceitado o projeto, e à equipe de Nutrição do Hospital, principalmente a Dra. Priscila Pinho, pela paciência e amparo na elaboração deste artigo.

\section{REFERÊNCIAS}

1. Stefanini E, Kasinski N, Carvalho AC. Guias de medicina ambulatorial e hospitalar de cardiologia. Cardiologia. São Paulo: Manole; 2004.

2. Mady C, lanni BM, Artega E, Cardiologia básica. São Paulo: Roca,1999.

3. Escott S. Nutrição relacionada ao diagnostico e tratamento. São Paulo: Manole 2011

4. Talma CP, Miranda ONB. Triagem nutricional em pediatria. Fundação Hospitalar do Estado de Minas Gerais, Diretrizes Clínicas Protocolos Clínicos; 2013.

5. Carvalho FC, Lopes CR, Vilela LC, Vieira MA, Rinaldi AEM, Crispim CA. Triagem e adaptação cultural da ferramenta Strong kids para a triagem do risco de desnutrição em crianças hospitalizadas. Rev Paul Pediatr 2013;31(2):159-65. doi.org/10.1590/S010305822013000200005

6. Bousquet LA, Stringhini MLF, Mortoza AS. Avaliação nutricional subjetiva global: instrumentos para triagem em crianças hospitalizadas. Rev. Aten. Saúde. São Caetano do Sul, v.14, n.47, p.67-74, jan./mar., 2016.

7. Aragão JA, Mendonça MP, Silva MS, Moreira NA, Sant' anna MEC, Reis FP. O Perfil Epidemiológico dos Pacientes com Cardiopatias Congênitas Submetidos à Cirurgia no Hospital do Coração. Rev Brasileira de Ciências da Saúde 2013; Vol17 (3): 263 -268. DOI:10.4034/RBCS.2013.17.03.08

8. Boeira G, Manfio F, Silva RM, lop S, Pedrotti T. Perfil nutricional de crianças hospitalizadas.UNIFRA Evento/Sepse, 2012 [Internet]. [Acesso em: 2016 Jul 20]. Disponível em: http://www.unifra.br/eventos/sepe2012/Trabalhos/6643.pdf

9. Secker DJ, Jeejeebhoy KN. Subjective global nutritional assessment for children. Am J Clin Nutr.2007;85:1083-9. [Acesso: em 21 de Janeiro 2017]. Disponível em: URL: https://www.ncbi.nlm.nih.gov/pubmed/17413109

10. Simões AP, Palchetti CZ, Patin RV, Mauri JF, Oliveira FLC. Estado nutricional de crianças e adolescentes hospitalizados em enfermaria de cirurgia pediátrica. Rev Paul Pediatr. 2010;28(1):41-7. [Acesso em 20 janeiro 2017] Disponível em: URL: http://www.scielo.br/scielo.php?script=sci_arttext\&pid=S0103-05822010000100008

11. Gouveia M. Validação Concomitante e preditiva de uma ferramenta de triagem de risco Nutricional em crianças Hospitalizadas. Dissertação (mestrado) - Universidade Federal de Pernambuco, CCS. Programa de Pós-Graduação em Saúde da Criança e do Adolescente. Recife, 2016.

12. Lama MRA, Moráis LA, Herrero ÁM, Chicano SC, Martinez RG, Ruzafa EL, et al. Validación de una herramienta de cribado nutricional para pacientes pediátricos hospitalizados. Nutr Hosp. 2012; 27(5):1429-1436. doi.org/10.3305/nh.2012.27.5.5467

13. Monteiro FPM, Oliveira CJ, Vitor AF, Araujo TL, Ximenes LB. Avaliação do estado nutricional de crianças com cardiopatia congênita sob a ótica de pender. Ver. Enferm. Vol 17: 581-588, out.-dez. 2009.

14. Araujo JSS, Régis CT, Gomes RGS, Silva CS, Abath CMB, Mourato FA, et al.Cardiopatia congênita no nordeste Brasileiro. Registro das cardiopatias congênitas na Paraíba, Rev. Bras Cardiol Vol.1; 13-19 PB, 2014. doi.org/10.5123/s1679-49742017000300011

15. Ribeiro IT, Silva LR, Mendes CMC, Coelho IB, Rocha SRF, Mattos AP, et al. Avaliação nutricional de crianças e adolescentes internados em um hospital privado de Salvador-Bahia. Rev. Ciênc. Méd. Biol., Salvador, v.14, n.1, p.5-9, jan./abr. 2015.

16. Teixeira G, Ribeiro A, Nascimento JS. Perfil Nutricional e Condição Sociodemográfica de Crianças e Adolescentes Portadores de Cardiopatia em Unidade Hospitalar do Recife-PE. Revista eletrônica,2015. [Acesso: 20 de Fevereiro 2017]. Disponível em: URL: https://reer.emnuvens.com.br/reer/article/view/5

17. Moutinho JCF. Estudo Rastreio de Risco Nutricional -strongkids das Crianças internadas no Hospital pediátrico de Coimbra. Dissertação (mestrado) - Faculdade de Medicina Universidade de Coimbra - FMUC. Coimbra, Portugal, 2014. 
18. Vallandro JP. Comparação de diferentes métodos de avaliação Nutricional não invasiva em Crianças Hospitalizadas. Tese (doutorado) - Universidade Federal do Rio Grande do Sul. Programa de Pós-graduação em saúde da criança e do adolescente. Porto alegre, 2016.

19. Marginean O, Pitea AM, Voidăzan S, Marginean C. Prevalence and Assessment of Malnutrition Risk among Hospitalized Children in Romania. J Health popul nutri ,2014 Mar;32(1):97-102.

\section{Colaboradores}

Todos os autores trabalharam em todas as etapas desde a concepção desta pesquisa até a revisão da versão final do artigo.

Conflito de Interesses: Os autores declaram não haver conflito de interesses.

Recebido: 17 de maio de 2019

Aceito: 14 de janeiro de 2020 